\title{
Disguised Electromagnetic Connections in Classical Electron Theory
}

\author{
Timothy H. Boyer \\ Department of Physics, City College of the City University \\ of New York, New York, New York 10031, USA
}

\begin{abstract}
In the first quarter of the 20th century, physicists were not aware of the existence of classical electromagnetic zero-point radiation nor of the importance of special relativity. Inclusion of these aspects allows classical electron theory to be extended beyond its 19th century successes. Here we review spherical electromagnetic radiation modes in a conducting-walled spherical cavity and connect these modes to classical electromagnetic zero-point radiation and to electromagnetic scale invariance. Then we turn to the scattering of radiation in classical electron theory within a simple approximation. We emphasize that, in steady-state, the interaction between matter and radiation is disguised so that the mechanical motion appears to occur without the emission of radiation, even though the particle motion is actually driven by classical electromagnetic radiation. It is pointed out that, for nonrelativistic particles, only the harmonic oscillator potential taken in the lowvelocity limit allows a consistent equilibrium with classical electromagnetic zero-point radiation. For relativistic particles, only the Coulomb potential is consistent with electrodynamics. The classical analysis places restrictions on the value of $e^{2} / \hbar c$.
\end{abstract}




\section{INTRODUCTION}

\section{A. Classical Electron Theory}

Classical electron theory was introduced at the end of the 19th century in the attempt to combine classical mechanics with classical electromagnetism. The theory, developed prominently by H. A. Lorentz, considered point charges (with the charge-to-mass ratio $e / \mathfrak{m}$ found by J. J. Thompson) in mechanical potentials and also interacting with electromagnetic radiation. The theory had a number of successes including providing explanations for the Faraday effect, optical birefringence, and the normal Zeeman effect, none of which involve Planck's constant $\hbar$. However, when combined with other contemporary ideas of physics, the theory led to unacceptable ideas such as the Rayleigh-Jeans law for thermal radiation equilibrium and to atomic collapse for the nuclear model of the hydrogen atom. In the first quarter of the 20th century, classical electron theory was gradually abandoned in favor initially of old quantum theory and then modern quantum mechanics. The transition to old quantum theory was described by Born in this way: "the stationary states of an atomic system shall be calculated, as far as possible, in accordance with the laws of classical mechanics, but the classical theory of radiation is disregarded." [1]

The "disregard" of radiation has occasionally caught the attention of classical-minded physicists. If one wishes to continue using classical electron theory to describe atomic phenomena, then the disregard of radiation represents an error. Indeed, classical electron theory can be extended beyond the successes of the very early 20th century by including two aspects which were not used by the physicists of that period: 1) the presence of random classical electromagnetic zero-point radiation, which involves Planck's constant $\hbar$, and 2) the importance of special relativity. These two neglected aspects allow classical theory to explain additional phenomena, including Casimir forces, van der Waals forces, the decrease of specific heats at low temperatures, diamagnetism, the Planck spectrum of thermal radiation, and the absence of atomic collapse.[2] [3]

\section{B. Classical Electromagnetic Zero-Point Radiation}

Many physicist are unfamiliar with the idea of classical electromagnetic zero-point radiation which provides a natural extension of traditional classical electron theory. [4] Classical 
electron theory consists of three basic ideas: 1) Maxwell's equations for the electromagnetic fields with point charges as sources, 2) Newton's second law for the motion of these charges due to forces, including forces due to electromagnetic fields, and 3) boundary conditions on the differential equations appearing in parts 1) and 2). Classical electromagnetic zero-point radiation appears in item 3) as the source-free boundary condition on the electromagnetic fields in Maxwell's equations; it corresponds to the electromagnetic fields which are already present when the field sources start to act. The typical texts of classical electromagnetism [5] [6] [7] [8] scarcely mention the source-free boundary conditions. Indeed, in our undergraduate laboratory experiments on electricity and magnetism, there is no mention of the radio waves and thermal radiation which are present in the room while the students adjust the sources for their experiments. However, at the level of atomic physics, the source-free fields may become important. Classical electromagnetic zero-point radiation is random classical radiation (like classical thermal radiation) with a Lorentz-invariant spectrum, $U_{z p}(\omega)=$ const $\times \omega$. This spectrum of energy $U$ versus frequency $\omega$ is also scale invariant, and so does not pick out a preferred length, or time, or energy; its presence is not immediately obvious. Zero-point radiation is regarded as the zero-temperature limit of blackbody radiation and can be treated with random phases just as was classical thermal radiation. At positive temperature, blackbody radiation has a different spectrum, and includes a special energy, length, and frequency which are evident in the Wien displacement rule $T \lambda=$ const. In order to match the experimentally observed Casimir forces due to random electromagnetic fields at low temperature, the scale of classical electromagnetic zero-point radiation is fixed so that $U_{z p}(\omega)=(1 / 2) \hbar \omega$ where $\hbar$ is Planck's constant. This place is the one point where Planck's constant $\hbar$ enters the extended version of classical electron theory.

\section{Disguised Electromagnetic Interactions}

It is a curious aspect of classical mechanical systems in classical electromagnetic zero-point energy that, in equilibrium, the mechanical system inherits the scale of motion involving $\hbar$ from the zero-point radiation, but the actual average motion is maintained as though there were no interaction with radiation. The interaction with zero-point radiation is disguised by the average balance between radiation emission and absorption. 
In the present article, we explore again the classical interaction between charged particles in potentials and classical zero-point radiation. Here we make use of spherical radiation modes, which will be unfamiliar to many physicists whose radiation training includes only plane waves, but we deal with much easier calculations involving not random radiation but rather coherent radiation scattering, and we deal with only circular particle orbits. We start with an adiabatically-invariant radiation spectrum in a spherical cavity, and then consider the interaction of this radiation with charged particles, especially charged particles in circular orbits. Adiabatic invariance for a mechanical system refers to a quantity which maintains its value under a slow change in one of the parameters of the system. For example, the ratio of energy $U$ to frequency $\omega$ for a harmonic oscillator is an adiabatic invariant $U / \omega=J$ under very slow changes of the oscillator frequency. The assumption of an adiabaticallyinvariant radiation spectrum mimics a crucial aspect of zero-point radiation. The situation of coherent radiation driving at the particle's resonant frequency removes the fluctuation aspects which are present in the full interaction with zero-point radiation. What remains is only the average values. However, even these average values are of interest and are indeed striking.

\section{Outline of the Article}

We start by discussing an adiabatically-invariant spectrum of classical radiation in a spherical, conducting-walled cavity. If the phases between the radiation modes are random, then such an adiabatically-invariant spectrum provides an approximation to classical zeropoint radiation, and, indeed, becomes Lorentz-invariant zero-point radiation in the largecavity limit. The zero-point radiation spectrum contains the least possible information since there is no basis for distinguishing any frequency; the spectrum has no preferred reference frame, and no preferred length or time or energy.

Next we turn to the interaction between classical electromagnetic radiation and a point charge in a potential. The familiar elementary case involves the scattering of a plane wave by a small linear electric dipole oscillator oriented along the $z$-axis. However, we simplify the situation to involve driving at the resonant frequency. Also, we point out that the plane wave can be expanded as a sum over spherical multipole modes, and the dipole scattering actually involves only the spherical electric multipole field of order $l=1, m=0$, the electric 
dipole field. All the rest of the plane wave is unscattered. The energy of the oscillator is determined by the energy of this one driving spherical multipole radiation mode. If we consider only this one spherical multipole field and the oscillating particle, then, in steadystate, the oscillator gives the false impression that it is a frictionless mechanical oscillation which has no interaction with radiation; the oscillatory motion continues but there is no average energy flow to or from the oscillator and no average flow of radiation energy in any direction.

Next we consider a nonrelativistic charged particle in a circular orbit in a classical central potential, driven (in dipole approximation) by an electric spherical multipole field of order $l=1, m=1$. Specification of the mechanical system requires information regarding the mass $\mathfrak{m}$ and charge $e$ of the particle and the parameters $\kappa$ and $\mathfrak{n}$ of the central potential taken as $V(r)=\kappa r^{\mathfrak{n}} / \mathfrak{n}$ where $\mathfrak{n}$ is a numerical constant. For charge $e$, the radiation field associated with a circular orbit depends upon only the radius $r$ and frequency $\omega$ of the mechanical orbit. Just as for the linear oscillator, the mechanical energy of the particle in the circular orbit is determined by the radiation energy of the driving electromagnetic field. We show that such a system is consistent with an adiabatically-invariant electromagnetic radiation spectrum only for the harmonic-oscillator potential, $V(r)=\kappa r^{2} / 2$, in the case of an orbit of small velocity.

In contrast with the nonrelativistic particle situation, a relativistic charged particle of arbitrary mass $\mathfrak{m}$ in a circular orbit of any non-zero radius in a Coulomb potential is indeed consistent with coherent electromagnetic radiation in an adiabatically-invariant spectrum. Finally, it is pointed out that steady-state motion for the classical charge puts limitations on the ratio $e^{2} /(\hbar c)$ involving the charge $e$ of the particle and the scale $\hbar$ of the driving zeropoint radiation. Classical electron theory starts at a more primitive level than quantum theory and so gives at least some restrictions on the value of the fine structure constant. Finally, we close with a brief discussion of the contrast in points of view of quantum theory and of classical electron theory with classical electromagnetic zero-point radiation. 


\section{MULTIPOLE RADIATION MODES IN A SPHERICAL CAVITY}

\section{A. Electric Fields of Spherical Radiation Modes}

A spherical cavity of radius $R$ in a perfect conductor allows standing radiation fields which are termed spherical electromagnetic field modes. For a cavity of finite radius $R$, the radiation modes are discrete and can be labeled by the discrete indices $n, l, m$. Although the spherical multipole radiation modes for arbitrary values of $l$ and $m$ are treated in the older, junior-level electromagnetism textbook of Slater and Frank, [5] only the dipole fields, $l=1$, appear in Griffiths' text, [6] and most students who encounter general spherical multipole fields do so in a graduate-level text such as Jackson[7] or Zangwill. [8] We are using Gaussian units throughout our analysis.

There are both electric and magnetic spherical multipole modes. The electric field inside the spherical cavity can be written as a sum over the multipole fields,

$$
\mathbf{E}(\mathbf{r}, t)=\operatorname{Re} \sum_{n=1}^{\infty} \sum_{l=1}^{\infty} \sum_{m=-l}^{m=l}\left(\mathbf{E}_{n l m}^{(E)}(\mathbf{r}, t)+\mathbf{E}_{n l m}^{(M)}(\mathbf{r}, t)\right) .
$$

The electric multipole fields $\mathbf{E}_{n l m}^{(E)}$ of (angular) frequency $\omega_{n l}^{E}=c k_{n l}^{E}$ are given by

$$
\mathbf{E}_{n l m}^{(E)}(\mathbf{r}, t)=\frac{a_{n l m}^{E}}{-i k_{n l}^{E}} \exp \left[-i c k_{n l}^{E} t\right] \nabla \times\left[j_{l}\left(k_{n l}^{E} r\right) \mathbf{X}_{l m}(\theta, \phi)\right]
$$

and the magnetic multipole fields $\mathbf{E}_{n l m}^{(M)}$ of frequency $\omega_{n l}^{M}=c k_{n l}^{M}$ are given by

$$
\mathbf{E}_{n l m}^{(M)}(\mathbf{r}, t)=a_{n l m}^{M} \exp \left[-i c k_{n l}^{M} t\right]\left[j_{l}\left(k_{n l}^{M} r\right) \mathbf{X}_{l m}(\theta, \phi)\right],
$$

where $a_{n l m}^{E}$ and $a_{n l m}^{M}$ are constants, $j_{l}$ is the spherical Bessel function of order $l$, and the vector spherical harmonic $X_{l m}$ is given by

$$
\mathbf{X}_{l m}(\theta, \phi)=\frac{1}{i \sqrt{l(l+1)}} \mathbf{r} \times \nabla Y_{l m}(\theta, \phi) .
$$

There are magnetic fields associated with the multipoles which can be written in analogous form, [7] but, for the radiation energy balance in our subsequent analysis, these fields are not needed.

\section{B. Frequencies of Standing Wave Modes in a Spherical Cavity}

The normal mode (angular) frequencies are given by $\omega=c k$ where wave numbers $k_{n l}^{M}$ and $k_{n l}^{E}$ are related to the zeros of the Bessel functions or derivatives of the Riccati-Bessel 
functions respectively [9]

$$
j_{l}\left(k_{n l}^{M} R\right)=0 \text { and }\left(\frac{d}{d r}\left[r j_{l}\left(k_{n l}^{E} r\right)\right]\right)_{r=R}=0 .
$$

If the radius $R$ of the conducting-walled cavity is sufficiently large compared to the frequency of interest so that $k_{n l} R>>l$, then the spherical Bessel functions can be approximated by their large-argument asymptotic forms

$$
j_{l}(x) \approx \frac{1}{x} \sin \left(x-\frac{l \pi}{2}\right)
$$

so that the zeros of the Bessel functions and of the Riccati-Bessel function can be approximated by

$$
k_{n l}^{M} \approx\left(n+\frac{l}{2}\right) \frac{\pi}{R} \text { and } k_{n l}^{E} \approx\left(n+\frac{l+1}{2}\right) \frac{\pi}{R}
$$

\section{Energy in Spherical Radiation Fields}

The energy in a spherical mode is connected to the expansion coefficients $a_{n l m}^{E}$ and $a_{n l m}^{M}$. The energy in a normal mode is equally divided (on time average) between the electric and magnetic fields. Thus taking the electric mode labeled by $n l m$, the energy for large $R$, $1<<k_{l m}^{E} R$, is

$$
\begin{aligned}
U_{n l m}^{(E)} & =\int d^{3} r \frac{1}{8 \pi}\left(E^{2}+B^{2}\right)=\frac{1}{4 \pi} \int_{0}^{R} d r r^{2} \int d \Omega \frac{1}{2} \mathbf{B} \cdot \mathbf{B}^{*} \\
& =\frac{\left|a_{n l m}^{E}\right|^{2}}{8 \pi} \int_{0}^{R} d r r^{2}\left[j_{l}\left(k_{n l}^{E} r\right)\right]^{2} \int d \Omega\left|\mathbf{X}_{l m}(\theta, \phi)\right|^{2} \approx \frac{\left|a_{n l m}^{E}\right|^{2} R}{16 \pi\left(k_{n l}^{E}\right)^{2}},
\end{aligned}
$$

where the large-argument asymptotic form in (6) has been used for the Bessel function and the standard normalization for the vector spherical harmonic.

For classical zero-point radiation, these expansion coefficients are chosen to correspond to an average energy $U=\hbar \omega / 2$ for each normal mode of (angular) frequency $\omega$. Then we have

$$
U_{n l m}^{(E)}=\frac{\left|a_{n l m}^{E}\right|^{2} R}{16 \pi\left(k_{n l}^{E}\right)^{2}}=\frac{1}{2} \hbar c k_{n l}^{E}
$$

or

$$
\left|a_{n l m}^{E}\right|^{2}=\frac{16 \pi\left(k_{n l}^{E}\right)^{2}}{R} U_{n l m}^{(E)}=8 \pi\left(k_{n l}^{E}\right)^{3} \frac{\hbar c}{R} .
$$

The energy in the magnetic multipole mode is analogous, $U_{n l m}^{(M)}=\hbar c k_{n l}^{M} / 2$. 


\section{Angular Momentum in Spherical Radiation Fields}

The electromagnetic fields in a spherical cavity carry not only energy but also angular momentum. For large values of $R$, spherical multipole fields have very specific ratios between energy and angular momentum in the radiation; for a spherical multipole field of order $l, m$, and frequency $\omega$, the ratio between the $z$-component of angular momentum $L_{z}$ and the energy $U$ in the multipole field in a large cavity is always given by [10]

$$
\frac{L_{z}}{U}=\frac{m}{\omega} .
$$

This restriction in the electromagnetic fields will then limit the charged-particle systems which can be coupled consistently to electromagnetic zero-point radiation.

\section{E. Scaling in Classical Electromagnetism}

The spectrum of electromagnetic zero-point radiation has no preferred length, or time, or energy, and so is invariant under an adiabatic compression or under a change of scale which maintains the values of the speed of light in vacuum $c$ and the charge of the electron $e$. This scaling aspect of classical electromagnetism which maintains the values of $c$ and $e$ often goes unrecognized, and represents a sharp break away from classical mechanics. Thus, classical mechanics contains no fundamental constants, and so mechanical systems can be chosen with arbitrary length dimensions, arbitrary periods of oscillation, and with arbitrary mass. In contrast, electromagnetic systems are sharply constrained. For example, a plane wave in vacuum of wavelength $\lambda$ is immediately known to have a frequency $c / \lambda$. This connection stands in contrast with that for a wave in a mechanical medium where a sound wave of known wavelength can have various velocities and frequencies depending upon the medium. Similarly, the electrostatic potential energy between two electrons separated by distance $r$ is immediately known to be $e^{2} / r$ whereas mechanical systems can have general potentials $V(\mathbf{r})$ connecting energy and separation.

Classical electromagnetic zero-point radiation (like all of electromagnetism) has been

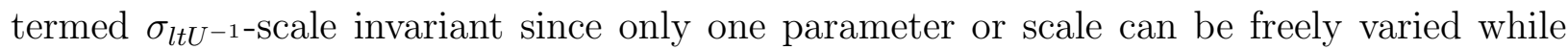

preserving the values of $c$ and e. [11] Thus, if we start with a spherical radiation mode labeled by $l$ and $m$ having frequency $\omega$ and energy $U$ in a spherical, conducting-walled cavity of radius $R$, and change the cavity radius $R$ adiabatically by a factor of $\sigma$ so that $R \rightarrow R^{\prime}=\sigma R$, 
then the spherical radiation mode has its frequency changed as $\omega \rightarrow \omega^{\prime}=\omega / \sigma$, and its energy

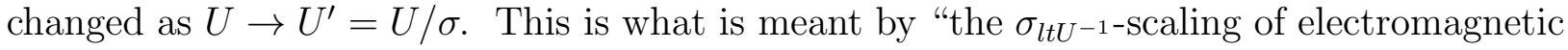
systems;" the lengths, times, and energies are all connected together under an adiabatic change or a change of scale. The situation of adiabatic invariance is probably most familiar in connection with slowly shortening the length of a mechanical simple pendulum by pulling the supporting string through a hole.[12] In the present case, we are dealing with a spherical radiation mode which has harmonic-oscillator behavior analogous to that of a small-amplitude pendulum. The spectrum of classical electromagnetic zero-point radiation

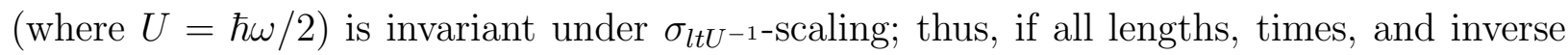
energies are transformed by the same factor $\sigma$, then the spectrum remains the same despite individual modes being changed in wave number, frequency, and energy. The individual modes are moved to new roles in the spectrum, but the spectrum is unchanged. The

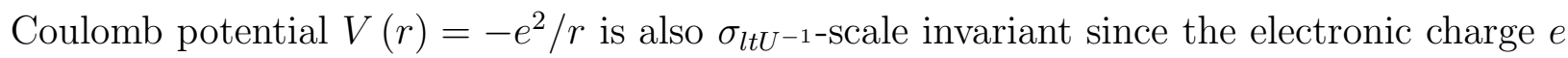
is an unchanged fundamental constant while the potential energy $V$ scales inversely as the length $r, V \rightarrow V^{\prime}=V / \sigma$ when $r \rightarrow r^{\prime}=\sigma r$.

\section{F. Forming an Adiabatically-Invariant Spectrum of Radiation}

All the radiation normal modes in a spherical conducting-walled cavity satisfy the conditions given in Eq. (5). We obtain an adiabatically invariant spectrum if we require that the energy for each mode is proportional to its frequency, $U=b \omega$, where $b$ is a single constant, the same for each mode in the cavity. In order to mimic zero-point radiation, we choose $b=\hbar / 2$, so that $U=b \omega=\hbar \omega / 2$. Under an adiabatic change in the cavity radius $R$, the ratio $U / \omega$ for each mode is preserved as a constant, and therefore the adiabatic change does not alter the proportionality constant in $U=b \omega=\hbar \omega / 2$. The frequency $\omega$ and the energy of each mode is changed, but the spectrum of radiation in the cavity is invariant under adiabatic change in the radius $R$. In the limit as the radius of the conducting cavity becomes ever larger, $R \rightarrow \infty$, the density of radiation normal modes become ever closer in frequency, and we arrive at a continuous, adiabatically-invariant spectrum of radiation. The relative phases of the radiation modes at different frequencies can be chosen randomly, and, in this fashion, we would obtain an adiabatically-invariant spectrum of random radiation mimicking classical electromagnetic zero-point radiation. In the limit $R \rightarrow \infty$, this 
radiation spectrum becomes Lorentz-invariant classical zero-point radiation.

\section{SCATTERING BY A SMALL ELECTRIC DIPOLE OSCILLATOR}

\section{A. Familiar Oscillator Example}

We start out with a familiar elementary example which emphasizes that particle oscillation can sometimes disguise the presence of the radiation which causes the oscillation. A familiar elementary scattering calculation[13] in classical electrodynamics treats a small, one-dimensional electric dipole oscillator $\mathbf{p}(t)=\widehat{z} e z(t)$, involving a particle of mass $\mathfrak{m}$ and charge $e$ in a harmonic potential $V(z)=\kappa z^{2} / 2$, oriented along the $z$-axis, and of natural frequency $\omega_{0}=\sqrt{\kappa / m}$, which is driven in dipole approximation by a plane wave of (angular) frequency $\omega$ traveling in the $x$-direction, $\mathbf{E}(x, y, z, t)=\widehat{z} E_{0} \cos [(\omega / c) x-\omega t]$. Newton's second law for the motion of the particle then gives

$$
\mathfrak{m} \ddot{z}=-\mathfrak{m} \omega_{0}^{2} z+\mathfrak{m} \tau \dddot{z}+e E_{0} \cos [\omega t]
$$

where the spring restoring force is $-\kappa z=-m \omega_{0}^{2} z$, and the radiation damping term involves the time $\tau=2 e^{2} /\left(3 \mathfrak{m} c^{3}\right)$. In steady-state motion, the electric dipole oscillates as

$$
z(t)=\operatorname{Re} \frac{(e / \mathfrak{m}) E_{0} \exp [-i \omega t]}{-\omega^{2}+\omega_{0}^{2}-i \tau \omega^{3}}=\operatorname{Re} \frac{(e / \mathfrak{m}) E_{0} \exp \left[-i \omega t+i \delta_{10}^{E}\right]}{\left[\left(-\omega^{2}+\omega_{0}^{2}\right)^{2}+\left(\tau \omega^{3}\right)^{2}\right]^{1 / 2}},
$$

where $\delta_{10}^{E}$ is the phase shift [14] between the incident and scattered waves with

$$
\tan \left(\delta_{10}^{E}\right)=\frac{\tau \omega^{3}}{\left(-\omega^{2}+\omega_{0}^{2}\right)}
$$

(Here the notation $\delta_{10}^{E}$ refers to the electric multipole mode of order $l=1, m=0$.)

\section{B. Driving the Oscillator at the Resonant Frequency}

If the oscillator is driven at resonance where $\omega=\omega_{0}$, then the particle mass $\mathfrak{m}$ cancels between the factor of $e / \mathfrak{m}$ in the numerator of Eq. (13) and the time $\tau$ in the denominator, the phase shift $\delta_{10}^{E}$ in Eq. (14) goes to $\pi / 2$, and the displacement becomes

$$
z(t)=\frac{3 c^{3} E_{0}}{2 e \omega_{0}^{3}} \sin \left[\omega_{0} t\right]
$$


The oscillation at $\sin \left[\omega_{0} t\right]$ is completely out of phase with the driving radiation $E_{0} \cos [\omega t]$. The amplitude of the oscillation is given by $z_{0}=3 c^{3} E_{0} /\left(2 e \omega_{0}^{3}\right)$. Although the emission and absorption of power are out of phase, the power emitted as radiation by the dipole oscillator is balanced over one oscillation cycle by the power delivered to the oscillator by the driving plane wave

$$
\left\langle\left[2 e^{2} /\left(3 c^{3}\right)\right] \omega_{0}^{4} z_{0}^{2} \sin ^{2}\left[\omega_{0} t\right]\right\rangle=\left\langle e z_{0} \omega_{0} E_{0} \cos ^{2}\left[\omega_{0} t\right]\right\rangle
$$

since $\left\langle\sin ^{2}\left[\omega_{0} t\right]\right\rangle=\left\langle\cos ^{2}\left[\omega_{0} t\right]\right\rangle=1 / 2$.

\section{Scattering Involves the Electric Spherical Radiation Mode $l=1, m=0$}

The plane wave $\mathbf{E}(x, y, z, t)=\widehat{z} E_{0} \cos [(\omega / c) x-\omega t]$ can be expanded in terms of spher-

ical multipole modes.[15] Although the traditional dipole scattering problem is phrased in terms of plane waves (which are familiar at the level of introductory physics and also appropriate for laboratory experiments), the interaction between the small electric dipole and radiation actually involves only the electric spherical multipole radiation mode $l=1, m=0$. Only the electric dipole multipole fields, $l=1, m=-1,0,1$, are finite in the limit as the displacement $r$ becomes very small, $r \rightarrow 0$, because, for small argument, the spherical Bessel functions go as the $l$ th power of the argument,

$$
j_{l}(x) \approx \frac{x^{l}}{(2 l+1) ! !} .
$$

For a small electric dipole oscillating along the z-axis, only the $\mathbf{E}_{l m}^{E}$ multipole given in Eq. (2) for $l=1, m=0$ will drive the oscillator. Thus, at the natural frequency of the dipole oscillator $\omega_{0}=c k_{n 1}^{E}$ and for $\mathbf{X}_{10}(\theta, \phi)=\widehat{\phi} i \sqrt{3 /(8 \pi)} \sin \theta$, this field becomes

$$
\begin{aligned}
\mathbf{E}_{10}^{E}(0, t) & =\operatorname{Re} \frac{a_{n 10}^{E}}{-i k_{n 1}^{E}} \exp \left[-i c k_{n 10}^{E} t\right] \nabla \times\left[\frac{k_{n 1}^{E} r}{3} \widehat{\phi} i \sqrt{\frac{3}{8 \pi}} \sin \theta\right] \\
& =-\widehat{z} \frac{a_{n 10}^{E}}{\sqrt{6 \pi}} \cos \omega_{0} t
\end{aligned}
$$

and is non-vanishing at the center of the dipole oscillation. This field agrees with driving by the plane wave when we take

$$
E_{0}=-\frac{a_{n 10}^{E}}{\sqrt{6 \pi}}=-\frac{1}{\sqrt{6 \pi}} \sqrt{\frac{16 \pi\left(k_{n l}^{E}\right)^{2}}{R} U_{n l m}^{(E)}}
$$


and the oscillator motion is given in Eq. (15). The oscillation amplitude of the small electric dipole is

$$
p_{0}=e z_{0}=\frac{3 c^{3}}{2 \omega_{0}^{3}} E_{0}=-\frac{3 c^{3}}{2 \omega_{0}^{3}} \frac{a_{n 10}^{E}}{\sqrt{6 \pi}} .
$$

\section{Interaction with a Single Radiation Mode}

We can imagine that the small electric dipole oscillator is located at the center of a spherical cavity with conducting walls containing an adiabatically-invariant spectrum of radiation, corresponding to that discussed earlier which forms a parallel with classical zeropoint radiation. For a cavity of finite radius $R$, the mass $\mathfrak{m}$ of the particle can be chosen so large that the line width $\Gamma=2 e^{2} \omega^{2} /\left(3 m c^{3}\right)$ is smaller than the separation in frequency between the discrete radiation modes of the cavity, and the particle interacts at its resonant frequency with a single mode of radiation which can be regarded as coherent radiation. As the radius $R$ increases toward infinity, the number of normal modes per unit frequency interval increases in compensation. In order to interact with a single frequency, the ratio $e / \mathfrak{m}$, involving the charge $e$ divided by the particle mass $\mathfrak{m}$, must become ever smaller as the radius $R$ of the cavity increases and the separation between the modes decreases.

\section{E. Analogue with Radiationless Stationary States}

If we consider the oscillator along the $z$-axis as driven, not by a plane wave, but by only the part of the plane wave with which it actually interacts, then we have a situation involving an oscillator in a standing spherical wave field $\mathbf{E}_{10}^{E}(\mathbf{r}, t)$. The electromagnetic wave forces the dipole into oscillation, and the oscillator then radiates into exactly the same spherical multipole mode, but with a phase shift of $\pi / 2$. In the steady-state situation, the energy provided to the oscillator exactly balances the energy emitted by the oscillator and gives a standing-wave radiation pattern. In this situation, no experiment would be able to detect an average flow of energy. There is an oscillating electromagnetic radiation field and also a charged particle oscillating in a harmonic potential. However, there is no average transfer of energy between the radiation and the charge. The oscillation amplitude of the small electric dipole is determined by the amplitude of the spherical multipole radiation field which maintains the amplitude of the oscillation against radiation emission by the 
oscillator. However, this driving is completely hidden by the radiation balance. This situation is a model for the sort of interaction with classical zero-point radiation which has been suggested[16] [17] as possibly providing a classical basis for the ground state of atomic hydrogen or of any quantum harmonic oscillator system.

\section{F. Consistency Under Adiabatic Change of Oscillator Frequency}

We have noted that zero-point radiation is adiabatically invariant. Here, it is of interest to note that the connection between the oscillator motion and the driving radiation is maintained during an adiabatic change of the oscillator frequency when the oscillator is in an adiabatically-invariant radiation spectrum. During an adiabatic change in the oscillator frequency, the ratio of the oscillator energy $\mathcal{E}$ to the oscillation frequency $\omega_{0}$ remains unchanged, $\mathcal{E} / \omega_{0}=$ const. But from Eqs. (20) and (9), the oscillator energy is related to the amplitude of the particle oscillation which is related to the radiation driving force, and so to the radiation energy per normal mode

$$
\mathcal{E}=\frac{1}{2} \mathfrak{m} \omega_{0}^{2} z_{0}^{2}=\frac{1}{2} \frac{\mathfrak{m}}{e^{2}} \omega_{0}^{2}\left(\frac{3 c^{3}}{2 \omega_{0}^{3}} \frac{a_{n l m}^{E}}{\sqrt{6 \pi}}\right)^{2}=\left[3\left(\frac{\mathfrak{m} c^{2}}{e^{2}}\right) \frac{c^{2}}{\omega_{0}^{2}} \frac{1}{R}\right] U .
$$

We will require that the factor of proportionality connecting the mechanical energy $\mathcal{E}$ to the radiation mode energy $U$ is always one,

$$
\left[3\left(\frac{\mathfrak{m} c^{2}}{e^{2}}\right) \frac{c^{2}}{\omega_{0}^{2}} \frac{1}{R}\right]=1 \text { or } \frac{1}{3}\left(\frac{e^{2}}{\mathfrak{m} c^{2}}\right) \frac{\omega^{2}}{c^{2}} R=1,
$$

so that the oscillator energy $\mathcal{E}$ equals the radiation mode energy $U$.

The situation here for single-mode driving is analogous to the narrow-line-width approximation $\tau \omega_{0}<<1$ involving random driving radiation. The quantity $\tau=2 e^{2} /\left(3 \mathfrak{m} c^{3}\right)$ gives a characteristic time associated with the charged particle, and the quantity $\Gamma=$ $(2 / 3)\left[e^{2} /\left(m c^{3}\right)\right] \omega_{0}^{2}$ gives a characteristic frequency spread. In this article, we consider a connection between the mechanical oscillator and a single radiation mode at the oscillator's resonant frequency. In earlier work with random radiation, we integrated over the sharply peaked function given in Eq. (13) to obtain the average energy of the oscillator $\langle\mathcal{E}\rangle$ as connected to the average energy of the radiation $\left\langle U\left(\omega_{0}\right)\right\rangle$ at the oscillator's resonant

frequency $\omega_{0} \cdot[16]$ [17] For large cavity radius $R$, the radiation modes are closely spaced so that $\Gamma$ would cover a large number of normal mode frequencies. Here we require that 
$e^{2} /\left(\mathfrak{m} c^{2}\right)$ goes to zero and $R$ goes to infinity in such a way that the condition (22) always holds. Then our one-mode-coherent-field calculations come close to the random zero-point calculations; the energy for the oscillator matches the energy per normal mode for the radiation modes at the resonant frequency of the oscillator. In both our resonant treatment and the traditional narrow-line-width calculation, the charge $e$ has entirely disappeared from the problem. There appears to be no connection between the mechanical system and the radiation system. Yet we have equality between the energy $U$ in the radiation field and the energy $\mathcal{E}$ of the mechanical system. The situation looks like a mechanics problem in the fashion suggested by Born's quote in the introduction where the radiation is to be "disregarded," except that here we know the amplitude of the mechanical oscillation is actually determined by the driving electromagnetic radiation.

\section{CIRCULAR PARTICLE ORBITS IN A CENTRAL POTENTIAL}

\section{A. Circular Orbits with Both Energy and Angular Momentum}

We now generalize the mechanical system under consideration. For the small onedimensional linear oscillator considered above, the oscillator energy is the one quantity which determines the amplitude of the oscillation, and, for a small electric dipole oscillator, this energy is determined by the amplitude of the driving coherent radiation at resonance. We now turn to charged particles in circular orbits in central potentials when driven by coherent radiation. Although our ultimate, long-term interest is the motion of charged particles in random classical zero-point radiation, the coherent-radiation situation can already remind us of important aspects of the connection between radiation and matter. A charged particle in a circular orbit in a central potential has both energy and angular momentum, which it must be radiating away. If the circular orbit is to be steady-state, then there must be electromagnetic driving fields which supply energy and angular momentum to the charge so as to balance the emitted energy and angular momentum.

\section{B. Circular Orbits for Nonrelativistic Particles}

Traditional classical electron theory treats nonrelativistic charged particles in arbitrary potential functions $V(\mathbf{r})$. Therefore, we turn to more general potential functions, but 
restrict our analysis to circular orbits in central potentials $V(r)$. We consider first charged particles of mass $\mathfrak{m}$ and charge $e$ in nonrelativistic circular orbits within a central potential $V(r)=\kappa r^{\mathfrak{n}} / \mathfrak{n}$. The particle motion lies in a plane which can be taken as the $x y$-plane, $\mathbf{r}(t)=r[\widehat{i} \cos (\omega t)+\widehat{j} \sin (\omega t)]$. For the mechanical motion of nonrelativistic particles in such potentials, the charge $e$ is regarded as irrelevant, and Newton's second law gives the balance for centripetal acceleration as

$$
\mathfrak{m} \frac{v^{2}}{r}=\kappa r^{n-1} \quad \text { or } \quad(\omega r)^{2}=v^{2}=\frac{\kappa}{\mathfrak{m}} r^{n} .
$$

Thus, in general, the ratio $\kappa / \mathfrak{m}$ provides the connection between the particle speed $v$ and the orbital radius $r$. The energy $\mathcal{E}$ of the particle in the circular orbit in the potential is given by

$$
\mathcal{E}=\frac{1}{2} \mathfrak{m} v^{2}+\frac{\kappa r^{\mathfrak{n}}}{\mathfrak{n}}=\left(\frac{n+2}{2 n}\right) \mathfrak{m} v^{2} .
$$

The angular momentum $J_{\phi}$ of the particle in its circular orbit is

$$
J_{\phi}=\mathfrak{m} r^{2} \omega=\frac{\mathfrak{m} v^{2}}{\omega}
$$

Then taking the ratio of the particle angular moment to energy, we have

$$
\frac{J_{\phi}}{\mathcal{E}}=\left(\frac{2 \mathfrak{n}}{\mathfrak{n}+2}\right) \frac{1}{\omega}
$$

Indeed, since we have the general relation $\omega=\partial \mathcal{E} / \partial J_{\phi}$, the product $J_{\phi} \omega$ of the orbital angular momentum and the angular frequency has the same dimensions as the energy $\mathcal{E}$. For our circular orbit in a central potential of the form $V(r)=\kappa r^{\mathfrak{n}} / \mathfrak{n}$, the ratio $J_{\phi} \omega / \mathcal{E}$ must be a dimensionless number. For our nonrelativistic particle, we find from Eq. (26),

$$
\frac{J_{\phi} \omega}{\mathcal{E}}=\left(\frac{2 \mathfrak{n}}{\mathfrak{n}+2}\right) \text {. }
$$

Thus for a nonrelativistic particle in a circular orbit, the ratio $J_{\phi} \omega / \mathcal{E}$ is a dimensionless number which is characteristic of the power $\mathfrak{n}$ of $r^{\mathfrak{n}}$ in the potential function $V(r)=\kappa r^{\mathfrak{n}} / \mathfrak{n}$.

\section{CONNECTION OF CIRCULAR PARTICLE ORBITS TO ELECTROMAG- NETIC RADIATION}

\section{A. Requirements on the Driving Radiation}

Since the particle in the potential $V(r)=\kappa r^{\mathfrak{n}} / \mathfrak{n}$ is assumed charged, we are interested in connecting our mechanical system with electromagnetic radiation. Electromagnetic ra- 
diation is a wave of speed $c$, so that we expect the nonrelativistic particle speed $\omega r=v$ to be much less than the radiation speed $c, v<<c$. Accordingly, the dipole approximation is appropriate for the connection between the nonrelativistic mechanical system and the radiation. The associated spherical radiation multipole coupled with this electromagnetic current is the electric multipole mode of order $l=1, m=1$, taken in the limit $\omega r / c=k r<<1$.

In a cavity of radius $R$ for a particle of sufficiently large mass $\mathfrak{m}$, the steady-state particle motion must be coupled to a single radiation mode of frequency $\omega=\omega_{n l m}$ for $l=1, m=1$, with the electric radiation field appropriately in phase with the particle orbital velocity so as to provide exactly the energy which is radiated away by the charged particle. Thus in this nonrelativistic dipole approximation, we have the radiation balance condition that

$$
\frac{2 e^{2}}{3 c^{3}} \omega^{4} r^{2}=e E_{0} \omega r
$$

or

$$
E_{0}=\frac{2 e}{3 c^{3}} \omega^{2} v
$$

for $v<<c$. In this case, we need the electric field $E_{0}=E_{11 \phi}^{(E)}$ for small displacement $r$. Then using Eq. (44) to obtain $X_{11}(\theta, \phi)=\sqrt{3 /(16 \pi)}(\widehat{\theta}+\widehat{\phi} i \cos \theta) \exp [i \phi]$, and substituting into Eq. (2) taken for small values of $r$, we find $E_{11 \phi}^{(E)}=a_{n l m}^{E} / \sqrt{12 \pi}$. Then combining Eqs. (29), (24), and (19), we have

$$
\begin{aligned}
\mathcal{E} & =\left(\frac{\mathfrak{n}+2}{2 \mathfrak{n}}\right) \mathfrak{m} v^{2}=\left(\frac{\mathfrak{n}+2}{2 \mathfrak{n}}\right) \mathfrak{m} \frac{3 c^{4}}{e^{2} \omega^{2}} \frac{U}{R} \\
& =\left(\frac{\mathfrak{n}+2}{2 \mathfrak{n}}\right)\left[3\left(\frac{\mathfrak{m} c^{2}}{e^{2}}\right) \frac{c^{2}}{\omega^{2}} \frac{1}{R}\right] U .
\end{aligned}
$$

Thus the mechanical energy of the particle in a circular orbit in the potential $V(r)=\kappa r^{\mathfrak{n}} / \mathfrak{n}$ is directly related to the energy $U$ of the driving radiation mode at the orbital frequency $\omega$. The connection between the mechanical and electromagnetic energies involves the same factor in square brackets which appeared for the linear oscillator in one dimension. If we apply the same condition (22) required for the one-dimensional oscillator motion, then here only the isotropic harmonic oscillator potential where $\mathfrak{n}=2$ has a mechanical energy $\mathcal{E}$ which equals the radiation energy $U$ in the driving mode.

The harmonic-oscillator potential is also singled out by the ratio $J_{\phi} \omega / \mathcal{E}$ in Eq. (27). This ratio agrees with the corresponding ratio $L_{z} \omega / U=1$ for the electromagnetic driving normal mode of order $l=1, m=1$ only when $\mathfrak{n}=2$, corresponding to the harmonic oscillator 
potential $V(r)=\kappa r^{2} / 2$,

$$
\frac{J_{\phi} \omega}{\mathcal{E}}=1 \text { (harmonic oscillator potential). }
$$

For a nonrelativistic particle in a Coulomb potential $V(r)=-e^{2} / r$ where $\mathfrak{n}=-1$, one finds

$$
\frac{J_{\phi} \omega}{\mathcal{E}}=-2 \quad \text { (Coulomb potential) }
$$

Thus, for the nonrelativistic charged particle in a Coulomb potential, the ratio in Eq. (32) does not agree with that of the associated driving radiation where $L_{z} \omega / U=1$.

\section{B. Radiation Equilibrium in Zero-Point Radiation}

We are interested not in the radiation equilibrium for a single mechanical system in some specialized radiation arrangement, but rather in the equilibrium of a general class of systems for varying mass $\mathfrak{m}$ in adiabatically invariant radiation. We emphasize that for a given charge $e$, the radiation equilibrium of an orbiting particle depends upon only the frequency $\omega$ and radius $r$ of the circular orbit or, equivalently, the speed $v$ and frequency $\omega$ of

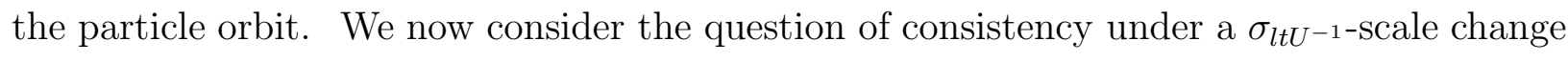
for both the particle orbit and the associated driving radiation. The radiation spectrum, which is assumed adiabatically-invariant, is unchanged under this scale change. However, while the speed $v$ of the nonrelativistic particle in its orbit and the angular momentum $J_{\phi}$ are both unchanged under this scale change, the frequency $\omega$ of the orbit will be changed by a factor of $1 / \sigma, \omega \rightarrow \omega^{\prime}=\omega / \sigma$. From Eq. (25), we see that

$$
v^{2}=\frac{J_{\phi} \omega}{\mathfrak{m}}
$$

This means that in order to retain the connection to an adiabatically-invariant radiation spectrum (where the length, time, and inverse energy all change together), the change in mass $\mathfrak{m}$ and the change in frequency $\omega$ must be connected. There are only two potentials for which this situation actually holds. Using equations (24) and (25), we see that the orbital frequency is connected to the particle mass $\mathfrak{m}$ and orbital angular momentum $J_{\phi}$ as

$$
\omega=\frac{\partial \mathcal{E}}{\partial J_{\phi}}=\frac{\kappa^{2 /(\mathfrak{n}+2)}}{\mathfrak{m}^{\mathfrak{n} /(\mathfrak{n}+2)}} J_{\phi}^{(\mathfrak{n}-2) /(\mathfrak{n}+2)} .
$$


Only for $\mathfrak{n}=-1$, corresponding to the Coulomb potential $V(r)=-e^{2} / r$, does the factor $\mathfrak{m}$ appear in first power in the numerator of Eq. (34). In this case, the constant $\kappa$ corresponds to the square of the electric charge, $\kappa=e^{2}$, and does not change under a $\sigma_{l t U^{-1} \text {-scale }}$ transformation, while

$$
v=e^{2} / J_{\phi} \quad(\text { Coulomb potential })
$$

which does not involve the mass $\mathfrak{m}$ at all. The particle energy is

$$
\mathcal{E}=-\frac{m e^{4}}{2 J_{\phi}^{2}} \quad \text { (Coulomb potential) }
$$

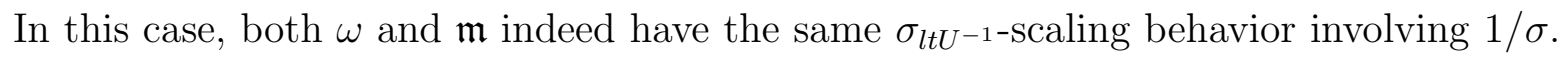

Although only the Coulomb potential allows a scaling of mass $\mathfrak{m}$ in agreement with the scaling of frequency $\omega$, the scaling situation can be avoided entirely by going to the largemass limit in Eq. (33). If the quantity $J_{\phi} \omega$ is fixed, the large-mass limit $\mathfrak{m} \rightarrow \infty$ will give the small-velocity limit $v \rightarrow 0$, which is indeed required for nonrelativistic particle behavior. But this is the situation for the harmonic oscillator potential $V(r)=\kappa r^{2} / 2$

where $\mathfrak{n}=2$, and the mechanical frequency is $\omega=\omega_{0}=\sqrt{\kappa / \mathfrak{m}}$. In this case, the spring constant $\kappa$ must increased along with $\mathfrak{m}$ so as to hold the frequency $\omega_{0}$ fixed. We see in Eq. (34) that the harmonic oscillator provides the only potential where the frequency is entirely independent of $J_{\phi}$.

\section{Successful Harmonic Oscillator Calculations}

Most of the calculations connecting charged mechanical systems to radiation involve linear oscillators in one spatial dimension. Indeed, Planck considered such an oscillator in random classical radiation at the end of the 19th century and concluded that the oscillator came to equilibrium with the radiation (in dipole approximation) when the energy of the oscillator matched the energy per normal mode of the random radiation at the frequency of the oscillator. It has been pointed out several times that adiabatic changes in the natural frequency of a linear harmonic oscillator [18] or adiabatic changes in the mechanical interaction frequencies between oscillators[19] will preserve the connection between the mechanical frequencies and classical electromagnetic zero-point radiation in the dipole approximation. All these one-dimensional calculations do not involve angular momentum. However, the isotropic oscillator in three dimensions was considered in connection with diamagnetism, 
and, indeed, the connection between the mechanical system and radiation was found to be preserved in classical electromagnetic zero-point radiation in the dipole approximation. [20]

The treatments of nonrelativistic charged particles in potentials other than the harmonic oscillator potential involve systems which will not fit consistently with classical electromagnetic zero-point radiation. Within classical physics, we must go to relativistic systems if we hope to move beyond the harmonic oscillator and its radiation connection at low velocity. Most physicists seem unaware of this requirement.

\section{RELATIVISTIC CHARGED PARTICLE IN THE COULOMB POTENTIAL}

\section{A. Relativistic Mechanical Motion}

The no-interaction theorem of Currie, Jordan, and Sudarshan[21] requires that any relativistic system involving an interaction between classical charged particles other than through point collisions requires the introduction of a field theory. The only familiar mechanical potential which can be extended to a fully relativistic system is the Coulomb potential which appears as part of classical electrodynamics. As noted above, only the

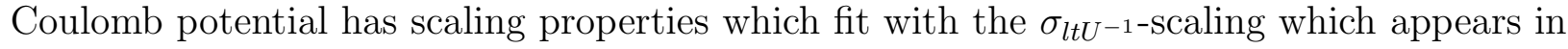
electromagnetic systems.

Once again, we consider a charged particle in a circular orbit in a potential, this time using relativistic analysis for the particle motion in the Coulomb potential $V(r)=-e^{2} / r$. In this case, the particle charge $e$ not only connects the particle to the radiation field, but also determines the particle motion in the Coulomb potential. Newton's second law for relativistic momentum gives for the force-balance of the centripetal acceleration

$$
\mathfrak{m} \gamma \frac{v^{2}}{r}=\frac{e^{2}}{r^{2}} \quad \text { or } \quad\left(\frac{e^{2}}{\mathfrak{m} c^{2}}\right) \frac{1}{r}=\gamma \beta^{2}
$$

where $\beta=v / c$ and $\gamma=\left[1-\beta^{2}\right]^{-1 / 2}$. Here the ratio $e^{2} /\left(\mathfrak{m} c^{2}\right)$ with the dimensions of length provides the connection between the velocity ratio $\beta$ contained in $\gamma \beta^{2}$ and the radius $r$. The energy of the particle in the circular orbit in the Coulomb potential is

$$
U_{e}=\mathfrak{m} \gamma c^{2}-\frac{e^{2}}{r}=\mathfrak{m} \gamma c^{2}-\mathfrak{m} \gamma v^{2}=\mathfrak{m} c^{2} \sqrt{1-\left(\frac{v^{2}}{c^{2}}\right)}=\mathfrak{m} c^{2} \sqrt{1-\beta^{2}}
$$


The angular momentum of the particle in the circular orbit is

$$
J_{\phi}=r \mathfrak{m} \gamma v=\frac{e^{2}}{v} \text { or } \quad \frac{v}{c}=\beta=\frac{e^{2}}{J_{\phi} c} .
$$

The relationship found here connecting the velocity $v$ to the angular momentum $J_{\phi}, v=$ $e^{2} / J_{\phi}$, holds for both relativistic and nonrelativistic treatments of a particle in a circular orbit in the Coulomb potential. The relativistic energy in the circular orbit can be rewritten as

$$
\frac{U_{e}}{\mathfrak{m} c^{2}}=\sqrt{1-\left(\frac{e^{2}}{J_{\phi} c}\right)^{2}}=\frac{1}{\gamma}
$$

giving the angular frequency

$$
\omega=\frac{\partial U}{\partial J_{\phi}}=\frac{\mathfrak{m} c^{3}}{e^{2}}\left(\frac{e^{2}}{J_{\phi} c}\right)^{3}\left[1-\left(\frac{e^{2}}{J_{\phi} c}\right)^{2}\right]^{-1 / 2},
$$

or

$$
\left(\frac{e^{2}}{\mathfrak{m} c^{3}}\right) \omega=\gamma \beta^{3} .
$$

Since $\omega r / c=\beta$, the equation (42) is consistent with Eq. (37). Thus the particle mass $\mathfrak{m}$ (when combined with the fundamental invariants $e$ and $c$ ) provides a scale for all the dimensions (length, time, and energy) connected to the mechanical motion of a relativistic particle in the Coulomb potential. Under a change in the particle mass $\mathfrak{m}$, this mechanical

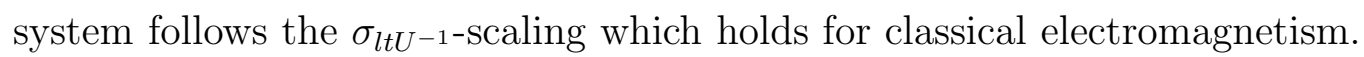

\section{B. Ratio of Angular Momentum to Energy}

Once again, we would like to calculate the ratio of angular momentum times angular frequency to energy for this relativistic situation. We find

$$
\frac{J_{\phi} \omega}{U}=\left(\frac{e^{2}}{J_{\phi} c}\right)^{2}\left[\sqrt{1-\left(\frac{e^{2}}{J_{\phi} c}\right)^{2}}\right]^{-2}=\frac{\beta^{2}}{1-\beta^{2}} .
$$

We notice that here for the relativistic Coulomb case, the ratio $J_{\phi} \omega / U$ is dimensionless, but, in sharp contrast with the nonrelativistic Coulomb case in (32) where $J_{\phi} \omega / U=-2$, is not a constant. Nonrelativistic mechanics contains no fundamental constants and the dimensionless ratio in Eq. (27) involves the dimensionless index $\mathfrak{n}$ which appears in the potential function $V(r)=\kappa r^{\mathfrak{n}} / \mathfrak{n}$. 
For a circular orbit of a relativistic particle in a Coulomb potential $V(r)=-e^{2} / r$, the ratio $J_{\phi} \omega / U$ in Eq. (43) can range between 0 and infinity, as the speed ratio $\beta=v / c$ ranges between 0 and 1. In the "nonrelativistic limit" of the relativistic expression, we would need the energy $\mathcal{E}$ above the rest energy $\mathfrak{m} c^{2}, \mathcal{E}=U-\mathfrak{m} c^{2}$, giving

$$
\begin{aligned}
\frac{J_{\phi} \omega}{\mathcal{E}} & =\left(\frac{J_{\phi} \omega}{U}\right) \frac{U}{\left(U-\mathfrak{m} c^{2}\right)}=\left(\frac{\beta^{2}}{1-\beta^{2}}\right) \frac{\left(1-\beta^{2}\right)^{1 / 2}}{\left[\left(1-\beta^{2}\right)^{1 / 2}-1\right]} \\
& =\beta^{2} \frac{1}{\left[-\beta^{2} / 2+\ldots\right]} \rightarrow-2 \text { for } \beta \rightarrow 0 .
\end{aligned}
$$

Thus in the limit of small particle speed $v$ compared to $c$, the expression (43) indeed goes over to the earlier result for a nonrelativistic particle in a Coulomb potential as given in Eq. (32).

For both a nonrelativistic particle and for a relativistic particle in a Coulomb potential, we find $v=e^{2} / J_{\phi}$. Therefore the speed $v$ is "small" only for $J_{\phi}$ "large." However, nonrelativistic mechanics contains no fundamental parameter involving speed, and all speeds are possible, $0 \leq v<\infty$. Similarly, there is no scale for angular momentum, and all values of angular momentum are possible. Thus, in nonrelativistic physics, there is no fundamental criterion for what is meant by a "small" or "large" speed. It is only when relativistic theory is introduced, either in terms of relativistic mechanics or in terms of relativistic radiation with the appearance of a fundamental speed $c$, that a criterion for a large or small speed appears. If the theory contains both the speed $c$ and the electronic charge $e$, then there is also a fundamental scale $e^{2} / c$ for angular momentum.

\section{Connection of a Relativistic Particle to Radiation}

A relativistic particle in a circular orbit in the Coulomb potential allows an entirely different connection to the radiation field from the situation for nonrelativistic particles. We are no longer restricted to small particle speeds in the isotropic harmonic oscillator potential coupled to radiation through the dipole approximation. Now radiation modes for

all values of $l$ and $m$ are allowed and indeed required. Burko[22] has given an analysis in terms of spherical multipole modes for the radiation emitted by a particle in uniform circular motion at any speed $v<c$. We can use his results, but the radiation pattern is now chosen as a standing wave pattern, rather than merely the emission of radiation as considered by 
Burko. Indeed, we may consider the radiation field as involving standing radiation modes in infinite space. Since the magnitude of the electronic charge $e$ is fixed, the dimensional units can now be scaled based upon the particle mass $\mathfrak{m}$, giving an energy ratio $U /\left(\mathfrak{m} c^{2}\right)$, a length ratio $r /\left[e^{2} /\left(\mathfrak{m} c^{2}\right)\right]$, and a time ratio $t /\left[e^{2} /\left(\mathfrak{m} c^{3}\right)\right]$. An adiabatic change in the invariant (rest) mass $\mathfrak{m}$ of the particle in the circular orbit will give a change in the energy $U$, the radius $r$, and frequency $\omega$ of the orbit, leaving the velocity of the particle unchanged. The change in the energy $U$, radius $r$, and frequency $\omega$ can all be compensated by an adiabatic change in the frequency of the driving radiation. However, if the driving radiation is adiabatically invariant, the spectrum is unchanged by any adiabatic change or any scale change. Both the mechanical system and the radiation involve the same one-parameter $\sigma_{l t U^{-1}}$-connection between the length, time, and energy under adiabatic changes. Indeed, the harmonic oscillator potential in the small-velocity limit and the relativistic Coulomb potential are the only classical scattering systems which preserve the spectrum of classical electromagnetic zero-point radiation. [18]

\section{RELATIVISTIC HYDROGEN AND LIMITS INVOLVING $e^{2} / c$ AND $\hbar$}

\section{A. Nonrelativistic Suggestions for the Hydrogen Ground State}

It has long been suggested that classical electromagnetic zero-point radiation might account for the ground state of hydrogen by providing radiation pick-up which balances the acceleration-related radiative energy loss. In 1975, a qualitative calculation[17] was presented based upon the radiative energy loss of a nonrelativistic charge in a circular orbit in a Coulomb potential

$$
\frac{d \mathcal{E}_{\text {loss }}}{d t}=\frac{2 e^{2}}{3 c^{3}} \omega^{4} r^{2}=\frac{2 e^{6}}{3 \mathfrak{m}^{2} c^{3} r^{4}}
$$

and the energy pick-up associated with a dipole rotor in classical zero-point radiation as

$$
\frac{d \mathcal{E}_{\text {gain }}}{d t}=\frac{e^{2} \hbar \omega^{3}}{2 \mathfrak{m c}^{3}}=\frac{e^{5} \hbar}{2 \mathfrak{m}^{5 / 2} c^{3} r^{9 / 2}}
$$

leading to an orbital radius $r=(3 / 4)^{2} \hbar^{2} /\left(m e^{2}\right)$. Although it was recognized at that time that use of pick-up by an electric dipole oscillator, in contrast to use of a dipole rotor, would give the exact result for the Bohr radius, the qualitative analysis clearly did not justify any suggestion of exact agreement. In 2021, a phase space calculation using action-angle 
variables for purely circular nonrelativistic Coulomb orbits in classical zero-point radiation suggested[23] a phase space probability distribution $P\left(J_{\phi}\right)=$ const $\times \exp \left[-J_{\phi} /(\hbar / 2)\right]$. However, such a phase space distribution will lead to a divergent result for the average nonrelativistic energy of the charged particle. Neither of these nonrelativistic calculations suggests any limit involving Planck's constant $\hbar$.

In nonrelativistic classical electromagnetic theory, Planck's constant $\hbar$ appears as the scale of zero-point radiation and may be chosen as large or as small as one desires. A nonrelativistic charged particle in a Coulomb potential $V(r)=-e^{2} / r$ involves only the mechanical parameters of charge $e$ and particle mass $\mathfrak{m}$. The presence of classical electromagnetic zero-point radiation introduces the constant $\hbar$ associated with the scale of the random radiation and having the dimensions of angular momentum. Thus the nonrelativistic classical theory of a charged particle in a Coulomb potential in zero-point radiation involves the fundamental parameters $e, \mathfrak{m}, \hbar$ and so allows exactly one length $\hbar^{2} /\left(\mathfrak{m} e^{2}\right)$, one time $\hbar^{3} /\left(\mathfrak{m} e^{4}\right)$, and one energy $\mathfrak{m} e^{4} / \hbar^{2}$. There exists no restriction on the values of $e$, $\mathfrak{m}$, or $\hbar$. In steady state, if such exists in nonrelativistic classical theory, we expect no restrictions on the average values of orbital radius, or frequency, or of energy. Rather, we should find actual values proportional to the corresponding length, time, and energy formed from $e, \mathfrak{m}$, and $\hbar$. And indeed the nonrelativistic qualitative calculations are consistent with this expectation.

\section{B. Relativistic Mechanics and a Lower Limit on the Value of $J_{\phi}$}

The situation involving a charge particle in a Coulomb potential is changed significantly by the introduction of relativity for the particle behavior. Now the mechanical system has parameters involving $e, \mathfrak{m}$, and $c$. Accordingly, from the mechanical parameters alone, we may form a length $e^{2} /\left(\mathfrak{m} c^{2}\right)$, a time $e^{2} /\left(\mathfrak{m} c^{3}\right)$, and an energy $\mathfrak{m} c^{2}$. Furthermore, we see from Eq. (40), that there is a limit on the smallest allowed value for the angular momentum $J_{\phi}$ of a relativistic particle in a Coulomb potential. Thus for our circular orbit, we must have $e^{2} / J_{\phi}=v<c$, or $e^{2} / c<J_{\phi}$. Indeed, for a general orbit (not necessarily circular) of a bound relativistic particle in a Coulomb potential, we have[24]

$$
\frac{U}{\mathfrak{m} c^{2}}=\left\{1+\left[\left(J_{3}-J_{2}\right) c / e^{2}+\sqrt{\left(J_{2} c / e^{2}\right)^{2}-1}\right]^{-2}\right\}^{-1 / 2},
$$


where the action variables are given by $J_{2}=J_{\phi}+J_{\theta}$, and $J_{3}=J_{2}+J_{r}$, and again there is the lower limit $e^{2} / c$ on the value of $J_{2}$ corresponding to the total angular momentum of the particle. We see that as the angular momentum decreases to $e^{2} / c, J_{\phi} \rightarrow e^{2} / c$ for the circular orbit, the particle speed in Eq. (39) increases to $c$, and the particle total energy in (40) decreases to zero . Thus, the situation for the relativistic particle is completely different from that of the nonrelativistic particle in Eqs. (35) and (36) where, as the angular momentum decreases to zero, $J_{\phi} \rightarrow 0$, the particle speed increases indefinitely, $v \rightarrow \infty$, while the particle energy decreases indefinitely, $\mathcal{E} \rightarrow-\infty$.

It is crucial to remember that the relativistic particle trajectories change dramatically when the value of the angular momentum gets near to $e^{2} / c$. [25] This aspect has nothing to do with Planck's constant $\hbar$ which appears as the scale of the adiabatic spectrum of classical zero-point radiation. Rather this aspect depends only upon the square-root of the quantity $J_{2}^{2}-\left(e^{2} / c\right)^{2}$ associated with the relativistic mechanical behavior of the particle in the Coulomb potential. For steady-state particle behavior, values of angular momentum $J_{2}$ smaller than $e^{2} / c$ are forbidden.

\section{Suggestion of Relations Relating $e^{2} / c$ and $\hbar$}

Now for our circular relativistic particle orbits described in Eqs. (37), and (39), the driving radiation must be such as to balance the relativistic radiation energy loss of the accelerating charge,

$$
P_{\text {loss }}=\frac{2 e^{2}}{3 c^{3}} \frac{\gamma^{4} v^{4}}{r^{2}}=\frac{2}{3}\left(m c^{2}\right)\left(\frac{m c^{3}}{e^{2}}\right)\left(\frac{e^{2}}{J_{\phi} c}\right)^{8}\left[1-\left(\frac{e^{2}}{J_{\phi} c}\right)^{2}\right]^{-3}
$$

where we require $e^{2} / c<J_{\phi}$. The energy gain, when treated as due to coherent radiation, corresponds to power delivered by the radiation $e v E_{\phi}(r, \phi, 0, t)$. However, in this case, we cannot assume that the radius $r$ of the orbit is small. As an approximation, we may take only the $l=1, m=1$ multipole field, giving

$$
P_{\text {gain }} \approx e v E_{11 \phi}^{(E)}(r, \phi, 0, t)=e c k r \sqrt{\frac{3}{16 \pi}} a_{11}^{E}\left\{\frac{1}{k r} \frac{\partial}{\partial r}\left[r j_{1}(k r)\right]\right\} .
$$

The crucial aspect remains that if the scale of the driving field given by $a_{11}^{E}$ is too small, then the driving radiation giving Eq. (49) cannot balance the radiation emitted according 
to Eq. (48). Thus if the scale $\hbar$ of the classical zero-point radiation were too small, say less than $e^{2} / c$, then no stable hydrogen atom would be possible in classical theory, since all the circular orbits would be in the forbidden region. A relativistic classical electromagnetic analysis seems to suggest limits on the value of the fine structure constant.

\section{CONTRASTING OUTLOOKS IN CLASSICAL AND QUANTUM THEO- RIES}

\section{A. Separate Behavior or Coupled Interactions Between Radiation and Matter?}

The classical point of view taken here stands in sharp contrast with the view appearing in quantum theory. In the classical view here, charged particle motion can be in steady state, despite emitting electromagnetic radiation, because of the presence of radiation which is driving the particle motion. However, only very specific potentials (the harmonic oscillator for a low-velocity particle and the Coulomb potential for a relativistic particle) allow a consistent treatment in classical electromagnetic zero-point radiation. In quantum theory on the other hand, quantum behavior is intrinsic for every system, and a mechanical particle can exist in its quantum ground state for any arbitrary potential $V(\mathbf{r})$. The particle state can be described by a time-independent wave function involving $\hbar$ even though the particle experiences no interactions at all with electromagnetic radiation.

The successes of 19th century classical electron theory, such as the Faraday effect, optical birefringence, and the normal Zeeman effect, do not involve the particle's interaction with any fundamental radiation distribution and do not involve Planck's constant $\hbar$. It is only with the introduction of classical electromagnetic zero-point radiation, that classical electron theory can be extended by detailed electromagnetic calculations to explain some microscopiclevel phenomena which do indeed involve Planck's constant $\hbar$, such as Casimir forces, van der Waals forces, the decrease of specific heats at low temperatures, diamagnetism, the Planck spectrum of thermal radiation, and the absence of atomic collapse.

As emphasized in the present article, the extended classical electron theory sharply restricts the allowed potential functions for charged particles in zero-point radiation. In general, the successful calculations of classical electron theory with classical electromagnetic zero-point radiation involve either free electromagnetic fields (such as for Casimir forces) or 
charged particles in harmonic potentials which are driven by classical zero-point radiation (such as for van der Waals forces). The classical calculations involving free electromagnetic fields are exactly parallel to the quantum counterparts; the average values for the energy per normal mode agree between the classical and the quantum theory, and this agreement is sufficient to account for the average Casimir forces.[17] On the other hand, for the interaction of dipole oscillator systems, the classical theory has the more primitive starting point, involving charged point particles in radiation. The classical theory leads to phase space distributions for the particles which have direct connections with the quantum harmonic-

oscillator probability distributions.[26] The classical and quantum theories agree regarding average values of forces but disagree regarding fluctuations. Although the average values of the forces (where there is agreement) have indeed been checked by experiment, the fluctuation aspects do not seem to have been tested.

The quantum point of view, which treats "quantum behavior" as something entirely different from classical charged particles interacting with zero-point radiation, sometimes gives rise to controversies. For example, some quantum physicists claim that Casimir forces between materials are due to quantum zero-point radiation while others insist that Casimir forces do not depend at all on quantum electromagnetic zero-point radiation but rather are van der Waals forces due to interactions arising from quantum charge fluctuations in the molecules of the materials. 27] From the viewpoint of classical electron theory, such controversies make little sense since there must be a consistent interaction between radiation and charged matter, and the same interaction gives rise to both Casimir forces and to van der Waals forces.

\section{B. Nonrelativistic or Relativistic Hydrogen Atom?}

The contrasting descriptions for charged particles in the Coulomb potential are also striking. For the Bohr model of old quantum theory, the Schroedinger wave function of nonrelativistic quantum theory, and Dirac's component wave function of relativistic quantum theory, the hydrogen ground state in quantum theory is complete without any need for electromagnetic radiation. This quantum viewpoint stands in sharp contrast with classical electron theory with classical electromagnetic zero-point radiation where the hydrogen ground state depends crucially upon the absorption of radiation energy from the classi- 
cal zero-point radiation; it is this energy absorption which prevents atomic collapse. For nonrelativistic classical electron theory including classical zero-point radiation, the necessary hydrogen calculations are incomplete but tantalizing, involving numerical simulations [3] and qualitative analytic calculations.[17] As suggested in the present article, only the full calculation of a relativistic particle in zero-point radiation is entirely consistent with classical theory. And such a calculation remains to be done. The relativistic treatment of a classical mechanical particle in a Coulomb potential includes novel aspects which remain unfamiliar to most physicists. [25] Such an analysis requires not the simplified model of the present article involving coherent radiation acting on the particle at resonance, but rather full random classical electromagnetic zero-point radiation with a scale set by $\hbar$ acting on relativistic charged particles. Indeed, even qualitative classical electromagnetic analysis suggests restrictions on the value of $e^{2} /(\hbar c)$. The classical electromagnetic treatment stands in complete contrast with quantum theory which assigns the same value $\hbar$ to every physical system, both mechanical and electromagnetic, and then changes the rules of interaction between radiation and matter so as avoid any restrictions on the value of the fine structure constant. It remains to be seen just how much information regarding the value of the fine structure constant will appear from a completely classical calculation.

\section{ACKNOWLEDGMENT}

The present article was stimulated by the work of Professor Daniel C. Cole suggesting the possibility of classical excited states based upon coherent classical radiation interacting with a classical nonrelativistic charged particle in an elliptical orbit in a Coulomb potential. D. C. Cole, "Subharmonic resonance and critical eccentricity for the classical hydrogen atomic system," Eur. Phys. J. D 72, 200-214 (2018). I wish to thank Professor Michael C. Mackey, Professor Brian R. La Cour, and Professor Daniel C. Cole for reading the manuscript and making helpful suggestions. 
[1] M. Born, The Mechanics of the Atom (Ungar, New York 1970), pp. 52-53.

[2] For example, see the reviews by T. H. Boyer, "Any classical description of nature requires classical electromagnetic zero-point radiation," Am. J. Phys. 79, 1163-1167 (2011); "Stochastic Electrodynamics: The Closest Classical Approximation to Quantum Theory,” Atoms 7(1), 2939 (2019).

[3] D. C. Cole and Y. Zou, "Quantum Mechanical Ground State of Hydrogen Obtained from Classical Electrodynamics," Phys. Lett. A 317, 14-20 (2003).

[4] T. H. Boyer, "Understanding zero-point energy in the context of classical electromagnetism," Eur. J. Phys. 37, 055206(14) (2016).

[5] J. C. Slater and N. H. Frank, Electromagnetism McGraw-Hill, New York 1947), Chapter XII.

[6] D. J. Griffiths, Introduction to Electrodynamics 4th ed. (Pearson, New York 2013), pp. 467-477.

[7] J. D. Jackson, Classical Electrodynamics 2nd ed. (John Wiley \& Sons, New York, 1975), Chapter 16.

[8] A. Zangwill, Modern Electrodynamics (Cambridge U. Press, 2013), Section 16.8.

[9] See ref. 5, p. 153.

[10] See ref. 7, p.750.

[11] T. H. Boyer, "Conformal Symmetry of Classical Electromagnetic Zero-Point Radiation," Found. Phys. 19, 349-365 (1989).

[12] See, for example, M. Born, Atomic Physics 7th ed. (Hafner, New York 1966), pp. 116-117.

[13] See, for example, A. Garg, Classical Electromagnetism in a Nutshell (Princeton U. Press, Princeton, NJ 08450, 2012), p. 508.

[14] See, for example, ref. 7, p. 773.

[15] See, for example, ref. 7, p. 769.

[16] T. W. Marshall, "Random electrodynamics," Proc. R. Soc. A276, 475-491 (1963); "Statistical Electrodynamics," Proc. Camb. Phil. Soc. 61, 537-546 (1965).

[17] T. H. Boyer, "Random electrodynamics: The theory of classical electrodynamics with classical electromagnetic zero-point radiation," Phys. Rev. D 11, 790-808 (1975); "General connection 
between random electrodynamics and quantum electrodynamics for free electromagnetic fields and for dipole oscillator systems," Phys. Rev. D 11, 809-830 (1975).

[18] See for example, T. H. Boyer, "Thermal Radiation Equilibrium: (Nonrelativistic) Classical Mechanics versus (Relativistic) Classical Electrodynamics," Eur. J. Phys. 42, 055102 (2021).

[19] D. C. Cole, "Derivation of the classical electromagnetic zero-point radiation spectrum via a classical thermodynamic operation involving van der Waals forces," Phys. Rev. A 42, 18471862 (1990).

[20] T. H. Boyer, "Diamagnetic behavior in random classical radiation," Am. J. Phys. 87,915-923 (2019).

[21] D. G. Currie, T. F. Jordan, and E. C. G. Sudarshan, "Relativistic Invariance and Hamiltonian theories of interacting particles," Rev. Mod. Phys. 35, 350-375 (1963).

[22] L. M. Burko, "Self-force approach to synchrotron radiation," Am. J. Phys. 68, 456-468 (2000).

[23] T. H. Boyer, "Relativity and radiation balance for the classical hydrogen atom in classical electromagnetic zero-point radiation," Eur. J. Phys. 42, 025205 (24pp) (2021).

[24] See, for example, H. Goldstein, Classical Mechanics 2nd ed. (Addison-Wesley, Reading, MA 1981), p. 498.

[25] T. H. Boyer, "Unfamiliar trajectories for a relativistic particle in a Kepler or Coulomb potential," Am. J. Phys. 75, 992-997 (2004).

[26] T. H. Boyer, "Dirac's Classical-Quantum Analogy for the Harmonic Oscillator: Classical Aspects in Thermal Radiation Including Zero-Point Radiation," Am. J. Phys. 88, 640-648 (2020).

[27] R. L. Jaffe, "Casimir effect and the quantum vacuum," Phys. Rev. D 72, 021301(R)(5) (2005); W. M. R. Simpson, "Ontological aspects of the Casimir effect," Studies in History and Philosophy of Modern Physics 48, 84-88 (2014); H. Nikolic, "Proof that Casimir force does not originate from vacuum energy," Phys. Lett. B 71, 197-202 (2016). I wish to thank Professor Michael Mackey for bringing the last two articles to my attention. 